\title{
GEOMETRY OF THE PRYTZ PLANIMETER
}

\author{
ROBERT L. Foote \\ Wabash College
}

\begin{abstract}
The Prytz planimeter is a simple example of a system governed by a nonholonomic constraint. It is unique among planimeters in that it measures something more subtle than area, combining the area, centroid and other moments of the region being measured, with weights depending on the length of the planimeter. As a tool for measuring area, it is most accurate for regions that are small relative to its length.

The configuration space of the planimeter is a non-principal circle bundle acted on by $S U(1,1)(\approx S L(2, \mathbb{R}))$. The motion of the planimeter is realized as parallel translation for a connection on this bundle and for a connection on a principal $S U(1,1)$-bundle. The holonomy group is $S U(1,1)$. As a consequence, the planimeter is an example of a system with a phase shift on the circle that is not a simple rotation.

There is a qualitative difference in the holonomy when tracing large regions as opposed to small ones. Generic elements of $S U(1,1)$ act on $S^{1}$ with two fixed points or with no fixed points. When tracing small regions, the holonomy acts without fixed points. Menzin's conjecture states (roughly) that if a planimeter of length $\ell$ traces the boundary of a region with area $A>\pi \ell^{2}$, then it exhibits an asymptotic behavior and the holonomy acts with two fixed points, one attracting and one repelling. This is obvious if the region is a disk, and intuitively plausible if the region is convex and $A \gg \pi \ell^{2}$. A proof of this conjecture is given for a special case, and the conjecture is shown to imply the isoperimetric inequality.
\end{abstract}

A planimeter is a mechanical instrument used to determine the area of a region in the plane. The user moves a "tracing point" around the boundary of the region. When the tracing point returns to its starting point, some feature of the instrument does not quite return to its initial position. This can be interpreted as holonomy, and is related to the area of the region.

Most planimeters consist of a rod, one end of which is the tracing point $T$. A wheel is attached to the rod that partially rolls and partially slides on the paper as the tracer point is moved. The most familiar such instrument is the polar planimeter (Figure 1(a)),

1991 Mathematics Subject Classification. 70F25, 53B15, 53A99, 53C65.

Key words and phrases. planimeter, non-holonomic, $S L(2, \mathbb{R})$, parallel translation, principal bundle, holonomy, phase shift, isoperimetric inequality.

The author would like to acknowledge helpful discussions on this topic with Felix Albrecht, Larry Bates, Richard Bishop, Lance Drager, and Jeff Lee.

To appear in Reports on Mathematical Physics. 
invented by Jacob Amsler in 1854, in which the end of the rod opposite the tracer point is hinged to a secondary rod, restricting its motion to a circle. In the linear planimeter (Figure 1(b)) the other end of the tracing rod is restricted to move along a linear track. The "roll" of the wheel is recorded on a scale, which reads out the area when the tracer point returns to its starting point. These work because the wheel mechanically integrates a 1-form that differs from $\frac{1}{2}(-y d x+x d y)$ by an exact form (see [Fo1] for details and other references).

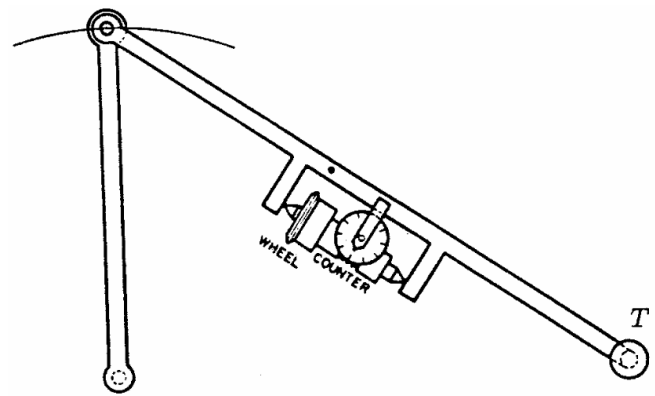

Figure 1(a). Polar Planimeter.

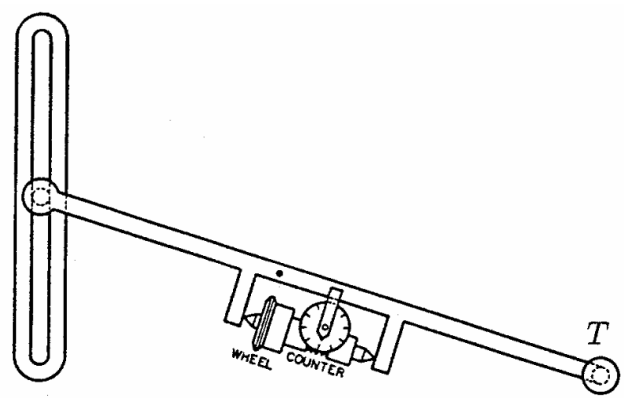

Figure 1(b). Linear Planimeter.

The Prytz planimeter (Figure 2), in contrast, contains no internally moving parts. It consists simply of a rod with its ends bent at right angles. One end, the tracer point $T$, is sharpened to a point. The other end, $C$, is sharpened to a chisel edge parallel to the rod. The chisel edge is usually slightly rounded, making it look similar to a hatchet, and consequently the device is also known as a "hatchet planimeter." It was invented in about 1875 by Holger Prytz, a Danish cavalry officer and mathematician, as an economical and simple alternative to Amsler's planimeter. Prytz referred to it as a "stang planimeter," "stang" being Danish for "rod." An amusing account of the history of the Prytz planimeter is given by Pedersen [Pe]. For a very complete history of planimeters through 1894, see Henrici [He].

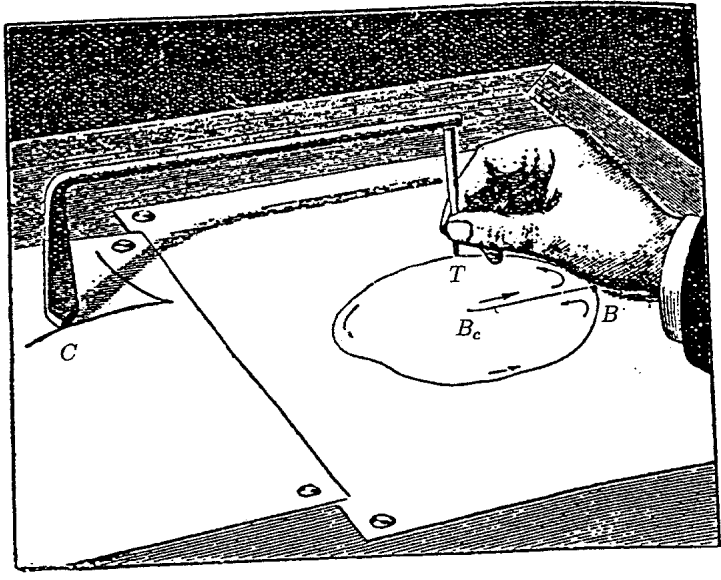

Figure 2. Prytz planimeter.

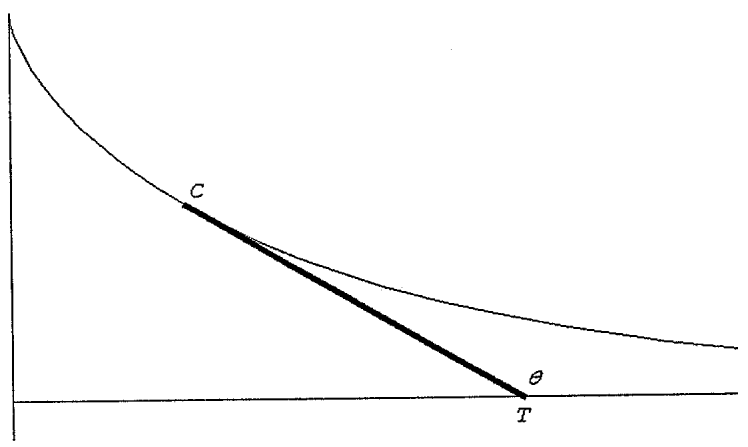

Figure 3. Standard tractrix. 
To use the planimeter, grasp it at the end with the tracer point, keeping its ends perpendicular to the plane. Move the tracer point along some curve taking care not to apply any torque to it. As $T$ moves along the curve, $C$ follows a pursuit curve that is always tangent to the rod. The paths of $T$ and $C$ are similar to those followed by the front and rear wheels of a bicycle. Note that if $T$ is moved along a straight line, the path of $C$ is a standard tractrix (Figure 3 ). Thus when $T$ moves along an arbitrary curve $\gamma$, the path of $C$ is called a tractrix of $\gamma$. Tractrices of various curves, particularly of circles, are considered in [L,Mo,Pou].

To measure the area of a region $\Omega$, start $T$ at some base point $B$ on $\partial \Omega$, and note the initial position of $C$. As $T$ moves around $\partial \Omega, C$ describes a zig-zag path (Figure 4 ), which Richard Bishop has likened to the motion of parking a car. When $T$ gets back to $B$, the chisel edge $C$ does not return to its starting point - it has undergone a displacement. Multiply the displacement $\sigma$ of $C$ by the length $\ell$ of the rod and you have the area. Actually it's only an approximation, but the error turns out to have a very nice geometric description.

We have implicitly assumed here that $\partial \Omega$ consists of a simple, closed curve, so $\Omega$ is bounded and simply connected. To precisely describe the behavior of the planimeter, we will also need to assume that $\partial \Omega$ is piecewise $C^{1}$. These assumptions about $\Omega$ will be made for the rest of the paper without further comment.

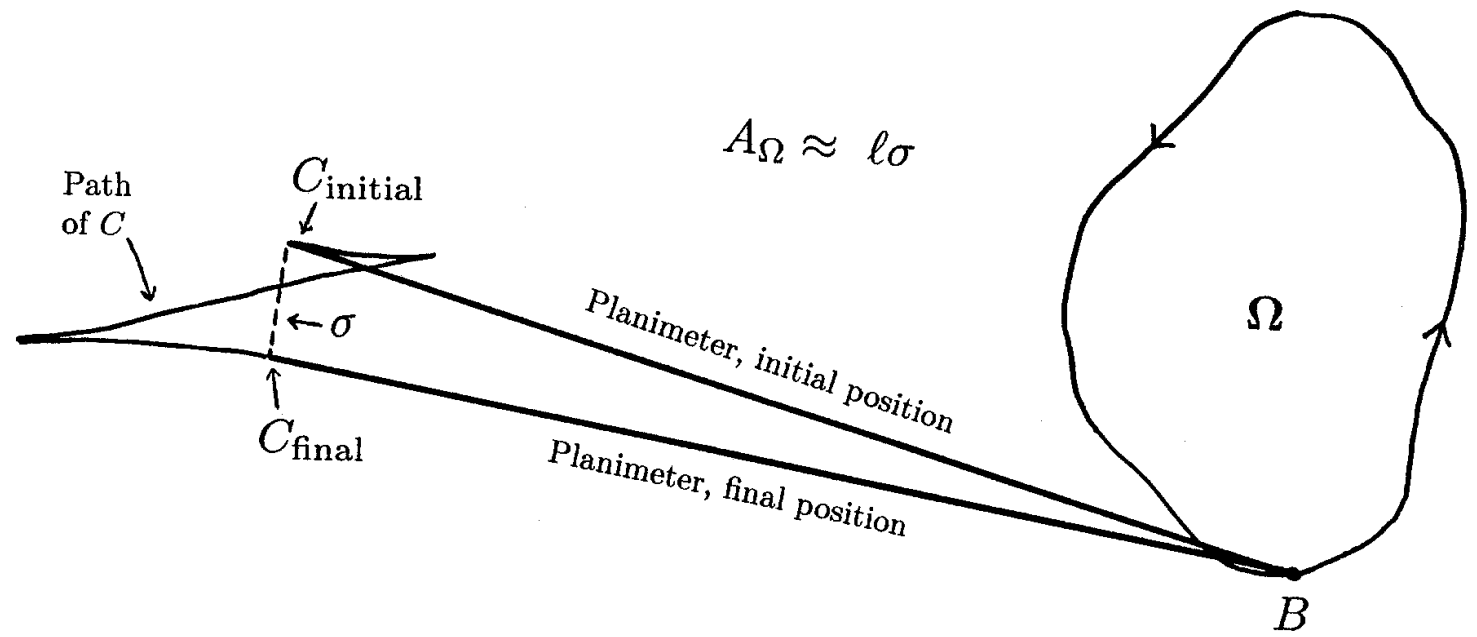

Figure 4. Motion of the Prytz planimeter.

Despite its simplicity and inherent inaccuracy as a measuring tool - in fact, because of its inherent inaccuracy - the Prytz planimeter is more interesting mathematically than the polar planimeter.

After giving the elementary theory of the Prytz planimeter, we will discuss Menzin's conjecture on how the planimeter behaves when tracing large regions and its connection to the isoperimetric inequality, the analytic approach of the inventor and one of his contemporaries, the behavior of the planimeter as a non-holonomic system-most specifically 
as parallel translation for a connection on a principal $S U(1,1)$-bundle and for the induced connection on an associated circle bundle - and finally, a proof of a special case of Menzin's conjecture.

\section{Elementary Theory}

Let $p$ and $q$ be distinct points in $\mathbb{R}^{2}$, and consider the segment joining them. Let $N$ be the unit vector perpendicular to the segment so that $q-p$ and $N$ form a positively-oriented frame.

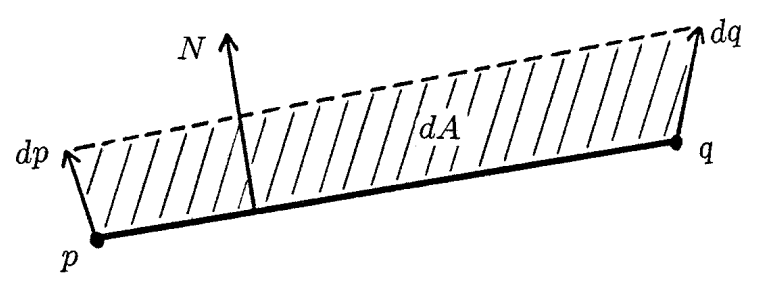

Figure 5 .

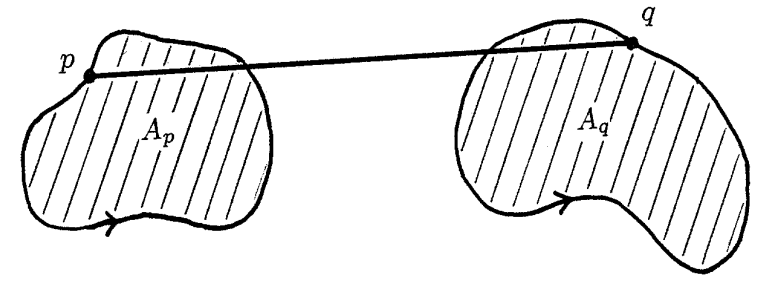

Figure 6 .

If the segment moves slightly, an infinitesimal oriented area is swept out (Figure 5). If $m=\frac{1}{2}(p+q)$ is the midpoint of the segment, then this area is given by $d A=\ell N \cdot d m=$ $\frac{1}{2} \ell N \cdot(d p+d q)$, where $\ell$ is the length of the segment (which can be variable), and $d p, d q$, and $d m$ are the infinitesimal displacements of $p, q$, and $m$, respectively. Note that $N$ points in the direction of motion for which the area is counted positively.

Let $d \sigma=N \cdot d p$ be the component of $d p$ in the direction of $N$. Noting that the infinitesimal rotation of the segment is $d \theta=\frac{1}{\ell} N \cdot(d q-d p)$, where $\theta$ is the angle of inclination of the segment relative to some fixed direction, the expression for the infinitesimal area can be written as $d A=\ell d \sigma+\frac{1}{2} \ell^{2} d \theta$.

These expressions for $d A$ are intuitively plausible. If $d p=d q$, then $d A=\ell N \cdot d m=$ $\frac{1}{2} \ell N \cdot(d p+d q)$ is just the area of a parallelogram. If $d p$ and $d q$ are parallel to the segment, they are orthogonal to $N$ and no area is swept out. If the segment rotates about its midpoint, then $d m=0$ and the oriented areas swept out by the two halves of the segment cancel. These motions account for all four dimensions of the configuration space of the moving segment, and so any infinitesimal motion of the segment is a linear combination of these. A similar intuitive argument can be made for $d A=\ell d \sigma+\frac{1}{2} \ell^{2} d \theta$. More rigorously, the expression $d A=\ell N \cdot d m$ is the integrand in the two-dimensional version of Guldin's formula for the area swept out by a moving segment [Cou,Fo1].

The formula for infinitesimal rotation (which will be needed later) is easily obtained by differentiating $N \cdot(p-q)=0$ and noting that $d N / d \theta$ is the unit vector $u=\frac{1}{\ell}(p-q)$. We get $0=u d \theta \cdot(p-q)+N \cdot(d p-d q)=\ell d \theta+N \cdot(d p-d q)$.

If the endpoints of the moving segment trace out curves, the expressions for $d A$ can be integrated, yielding the total oriented area swept out. In particular, if the endpoints trace out closed curves (Figure 6), it is easy to show [Fo1] that the oriented area swept out is 
$A_{q}-A_{p}$, where $A_{p}$ and $A_{q}$ are the oriented areas enclosed by curves traced out by $p$ and $q$, respectively.

The moving segment becomes a Prytz planimeter if we fix its length $\ell$ and use the endpoint $q$ as the tracer point $T$. The behavior of the planimeter is then given simply by the non-holonomic restriction $d \sigma=0$, that is, the infinitesimal movement of the chisel point $C$ has no component in the direction of $N$. As a consequence, $d A=\frac{1}{2} \ell^{2} d \theta$ and the total oriented area swept out by the planimeter at any moment is $A=\frac{1}{2} \ell^{2} \Delta \theta$, where $\Delta \theta$ is the net rotation of the planimeter. This gives a simple proof that the area under the standard tractrix (Figure 3 ) is $\frac{1}{4} \pi \ell^{2}$.

Now consider what happens when the tracer point is moved around the boundary of a region $\Omega$ in the positive direction, starting and ending at some base point $B \in \partial \Omega$ (Figure 4). The chisel point $C$ does not return to its original position. For regions that aren't too large relative to $\ell$ (and for which the planimeter gives the most accurate results, as we will see), the chisel point comes to rest at a point close to its original position. Now imagine the user fixing the tracer point $T$ at $B$ and rotating the planimeter about $B$ (this motion violates the non-holonomic constraint) so that $C$ moves along a circle back to its initial position (we will call this circle - with center $B$ and radius $\ell$ - the "initial circle"). As both endpoints would now have traced out closed curves, the oriented area swept out is $A_{q}-A_{p}=A_{\Omega}-A_{\gamma}$, where $A_{\Omega}$ is the area of $\Omega$ and $A_{\gamma}$ is the oriented area enclosed by the curve $\gamma$ formed by the zig-zag path of $C$ during the normal use of the planimeter plus the arc of the initial circle from the final position of $C$ back to its initial position. The oriented area $A_{q}-A_{p}$ also equals the integral of $d A=\ell d \sigma+\frac{1}{2} \ell^{2} d \theta$. As the initial and final angles of the planimeter are the same, $d \theta$ integrates to 0 . Along the part of $\gamma$ followed by the chisel we have $d \sigma=0$, but along the part of $\gamma$ that is the arc of the initial circle, $d \sigma$ integrates to $\sigma$, the length of that arc. Thus we have

$$
A_{\Omega}-A_{\gamma}=\ell \sigma, \quad \text { or } \quad A_{\Omega}=\ell \sigma+A_{\gamma},
$$

and we see that the error made by the approximation $A_{\Omega} \approx \ell \sigma$ is $A_{\gamma}$. In a typical use of the planimeter, the curve $\gamma$ encloses a number of "triangular" regions. These regions are generally small compared to $\Omega$. Moreover the boundaries of those regions inside the initial circle are traced with the opposite orientation of those outside, and so their oriented areas have opposite signs in their sum $A_{\gamma}$. Keeping this in mind, a good starting position is one that will result in the chisel edge spending part of its time inside the initial circle and part of its time outside. For example, one could start with the planimeter perpendicular to a line that roughly bisects the region.

Another way to minimize the error, suggested by several authors $[\mathrm{Ba}, \mathrm{Cr}, \mathrm{Hi}, \mathrm{K}, \mathrm{Pr} 1-$ $4, \mathrm{Sa}, \mathrm{St}]$, is to draw a line segment from $B$ to the centroid $B_{c}$ of the region. Instead of starting the tracer point at $B$, start at $B_{c}$. Trace along the segment $B B_{c}$ to $B$, then 
around the curve, and finally back along $B B_{c}$, stopping at $B_{c}$, as suggested in Figure 2 . This, of course, leads to the problem of locating the centroid, which is at least as hard as computing the area! In practice, one simply guesses. Prytz and Hill are the only authors who give any argument that the centroid should be used as the base point (outlined in Section 3). The other authors simply appeal to this as a geometrically plausible way to balance the triangular regions inside and outside the initial circle.

Similar arguments can be made to explain how other planimeters work, and have appeared in a variety of forms dating from the early 1800's (see [Fo1] for references). The argument given above to explain the behavior of the Prytz planimeter in particular is due to Henrici [He], who was the first to give a common theoretical setting for most of the planimeters invented up to 1894. This argument also appears in a paper by Kriloff [K], and may have been discovered independently by him.

It is interesting that this simple geometric argument can be made without referring to the exact relationship between the movement of the tracer point and the angle $\theta$ between the planimeter rod and some fixed direction. This relationship is the focus of much of the rest of the paper. Recall the expression for the infinitesimal rotation of a moving line segment, $\ell d \theta=N \cdot(d q-d p)$, which for the planimeter becomes $\ell d \theta=N \cdot d q$, since $d \sigma=N \cdot d p=0$. If the tracer point $T=q$ follows the curve $\gamma: \mathbb{R} \rightarrow \mathbb{R}^{2}$, the differential equation governing $\theta$ is then $\ell \frac{d \theta}{d t}=N \cdot \gamma^{\prime}(t)$. If $\theta$ measures the angle between the planimeter and the $x$-axis, i.e., $p-q=\ell(\cos \theta \mathbf{i}+\sin \theta \mathbf{j})$, the equation becomes $\ell \frac{d \theta}{d t}=\sin \theta \frac{d x}{d t}-\cos \theta \frac{d y}{d t}$. If the tracer point moves along the $x$-axis with $x=t$, as in Figure 3 , we get $\ell \frac{d \theta}{d x}=\sin \theta$, the solution of which is easily seen to be

$$
\tan \frac{\theta}{2}=A e^{x / \ell}
$$

This can be rewritten as $\tan \theta=-\operatorname{csch}(a+x / \ell)$, where $a=\log A$. If the planimeter starts perpendicular to the $x$-axis when $x=0$, then $a=0$, and $\operatorname{so} \tan \theta=-\operatorname{csch}(x / \ell)$. This is the slope of the line tangent to the tractrix when that curve is parameterized by the point where the tangent meets the axis (see, e.g., [Cox]):

$$
\tau(x)=(x-\ell \tanh (x / \ell), \ell \operatorname{sech}(x / \ell)) .
$$

Variations of the Prytz planimeter have been made, the most notable ones being by Goodman and Scott. As we have seen, the length of the arc along the initial circle between the initial and final locations of the chisel edge is the important quantity to measure, and not simply the distance between these points, although the latter is usually adequate. The variations due to Goodman and Scott allow the direct measurement of this arc. Goodman $[\mathrm{G}]$ incorporated a curved scale with radius $\ell$ into the planimeter rod, so the scale lies along the initial circle when applied to the points (Figure 7). Scott's variation [Sc] implements 
the idea in the discussion above that brings the chisel end of the planimeter back to its initial position. He put a wheel next to the chisel edge with its axis parallel to the rod. This wheel does not contact the paper during the tracing of $\partial \Omega$, but rides slightly above it. When the tracing point returns to the base point, the planimeter is tilted slightly, bringing the wheel into contact with the paper. The user then rotates the planimeter around the tracer point. The wheel rolls along the initial circle between the points, measuring the arclength. Both inventors, particularly Scott, seemed to believe that they were addressing the cause of the instrument's error, but the proof above and the analysis by Prytz and Hill in Section 3 show that the error is more complicated (and more interesting) than this.

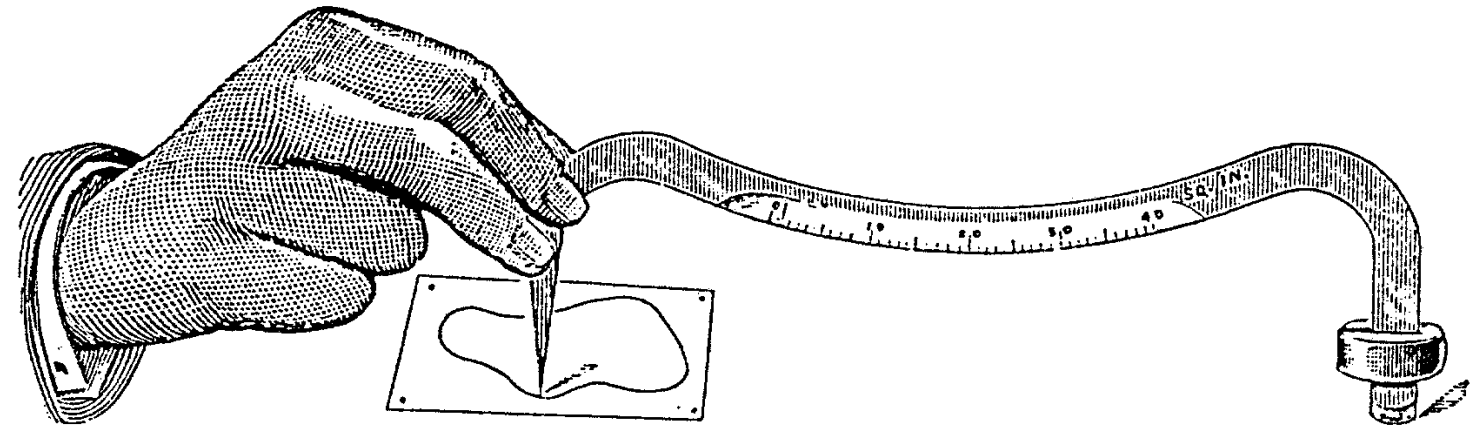

Figure 7. Goodman's planimeter.

The planimeters of Prytz, Goodman, and Scott were all marketed (the original Prytz planimeter was produced by the firm of Knudsen in Copenhagen [Pe]). The additional details of the latter two, however, defeated Prytz's original purpose of economy relative to Amsler's planimeter. In reaction to Goodman and Scott, Prytz [Pr3] advised engineers "rather than use the 'improved stang planimeters,' let a country blacksmith make them a copy of the original instrument." A collector has sent the author two photographs, one of a Prytz planimeter manufactured in the Netherlands and one of a Goodman planimeter, which are posted on the author's web page (URL at the end of this paper). The author would be very interested to learn of other instruments of this type that still exist.

\section{Menzin's Conjecture And the Isoperimetric Inequality}

When the planimeter traces the boundary of a polygonal region $\Omega$ with edges that are large compared to $\ell$, the length of the planimeter, it is intuitively clear that the path of $C$ asymptotically approaches a particular tractrix that is closed, and that when the planimeter follows this closed tractrix, it makes a complete rotation. This also happens when $\Omega$ is a disk of radius $R>\ell$; it is easily shown that the closed tractrix is a circle of radius $\sqrt{R^{2}-\ell^{2}}$. Furthermore, it is intuitively plausible for large convex regions. After much experimentation, Menzin [Me] conjectured that this happens whenever $A_{\Omega}>\pi \ell^{2}$. The last section contains a proof of Menzin's conjecture when $\partial \Omega$ is a parallelogram. 
In spite of its title (having to do with Amsler's planimeter), the paper by Morley [Mo] goes into considerable detail on the tractrices of circles. In addition to the case where $R>\ell$, he notes that when $R=\ell$ the asymptotic tractrix reduces to the center of the circle, but it is attractive only from one side. When $R<\ell$ each tractrix is made up of regularly spaced cusps.

It turns out that Menzin's conjecture implies the isoperimetric inequality. To see this, note that when $C$ follows the closed tractrix, $d A=\ell d \sigma+\frac{1}{2} \ell^{2} d \theta$ integrates to $\pi \ell^{2}$, since $d \sigma=0$ and the planimeter makes a complete rotation. Since $C$ traces a closed path, the total area swept out is $A_{\Omega}-A_{C}$, where $A_{C}$ is the area enclosed by the path of $C$ (which does not include an arc of the initial circle). Furthermore, note that $d A$ can be written as $d A=\ell d \sigma_{T}-\frac{1}{2} \ell^{2} d \theta$, where $d \sigma_{T}=N \cdot d q$ is the component of $d q$ (the infinitesimal displacement of the tracer point) in the direction of $N$. This expression for $d A$ integrates to $\ell \sigma_{T}-\pi \ell^{2}$, where $\sigma_{T}=\int d \sigma_{T}$. (Note that if a wheel is mounted on the rod at $T$ similar to the wheel on a polar or linear planimeter, then $\sigma_{T}$ is the total signed distance the wheel rolls.) Thus we have

$$
\pi \ell^{2}=A_{\Omega}-A_{C}=\ell \sigma_{T}-\pi \ell^{2}
$$

By Menzin's conjecture this holds under the assumption that $A_{\Omega}>\pi \ell^{2}$. In the limiting case where $A_{\Omega}=\pi \ell^{2}$, we have $A_{C}=0$. A little algebra yields $\sigma_{T}^{2}=4 \pi A_{\Omega}$. Noting that $d \sigma_{T}$ measures only a component of the infinitesimal distance the tracer point moves $\left(d \sigma_{T} \leq d s\right)$, we have $\sigma_{T} \leq L_{\partial \Omega}$, where $L_{\partial \Omega}$ is the length of $\partial \Omega$. The isoperimetric inequality follows:

$$
L_{\partial \Omega}^{2} \geq \sigma_{T}^{2}=4 \pi A_{\Omega}
$$

Furthermore, $L_{\partial \Omega}^{2}=4 \pi A_{\Omega}$ implies $d \sigma_{T}=d s$, that is, the tracer point only moves in the direction perpendicular to the rod. In this case the chisel edge does not move at all, and the tracer point describes a circle of radius $\ell$. Thus the isoperimetric inequality is an equality only if $\partial \Omega$ is a circle.

For other connections between planimeters and isoperimetric inequalities, see [Fo1\&2].

\section{Analysis by Prytz And Hill}

Prytz and Hill studied the "stang" planimeter analytically, rather than geometrically. Prytz's original paper [Pr1] was published anonymously in Danish under the pseudonym 'Z' (see $[\mathrm{Pe}]$ for the story behind this). His subsequent short notes on this are very tersely written [Pr2\&4]. Hill's account is much more readable [Hi]. 


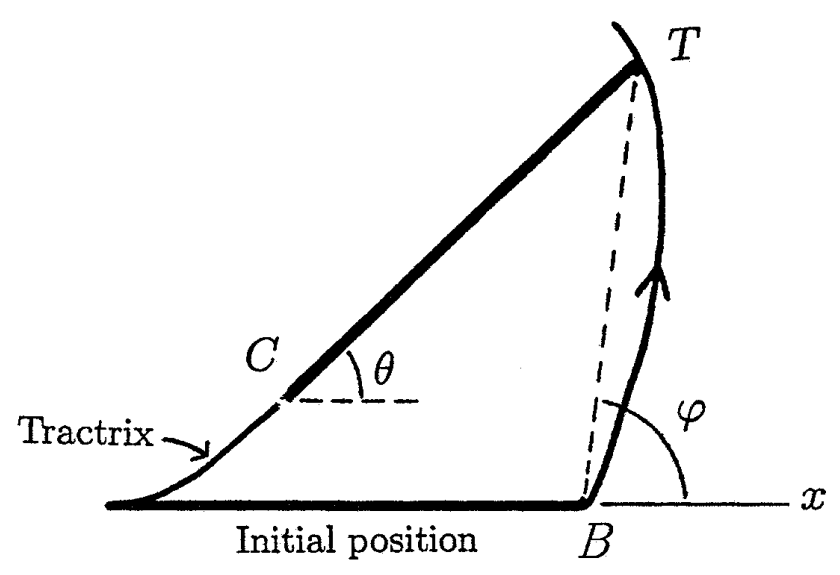

Figure 8.

Both authors use (1) to write an infinite series expansion for the rate at which $\theta$ changes when the tracer point moves along an arbitrary curve (Figure 8). Hill, who keeps more terms, gets

$$
\ell^{2} \frac{d \theta}{d \varphi}=\left(\frac{r^{2}}{2}+\frac{r^{4}}{8 \ell^{2}}+\frac{r^{6}}{144 \ell^{4}}+\ldots\right)+\left(\frac{r^{3}}{3 \ell}+\frac{r^{5}}{30 \ell^{3}}+\ldots\right) \cos (\varphi-\theta) .
$$

Here $(r, \varphi)$ are polar coordinates of the tracer point $T$ about the base point $B$.

Their analysis depends on the function $r / \ell$ being small on the path followed by the tracer point, which is to say, that $\Omega$ is small and that the base point $B$ is not far from $\Omega$, both relative to $\ell$, the length of the planimeter. As the tracer point moves around $\partial \Omega$, the dominant terms not depending on $\theta$ integrate to

$$
\int_{\partial \Omega} \frac{r^{2}}{2} d \varphi=A \quad \text { and } \quad \int_{\partial \Omega} \frac{r^{4}}{8 \ell^{2}} d \varphi=\frac{1}{2 \ell^{2}} \int_{\Omega} r^{2} d A=\frac{I_{B}}{2 \ell^{2}},
$$

where $A$ is the area of $\Omega$ and $I_{B}$ is the second moment (the moment of inertia) of $\Omega$ about $B$.

Assuming the initial direction, $\theta=0$, of the planimeter is the positive $x$-axis, and that $|\theta|$ stays small (which follows from the assumption that $r / \ell$ is small), Hill writes the remaining most dominant term as

$$
\frac{1}{3 \ell} \int_{\partial \Omega} r^{3} \cos (\varphi-\theta) d \varphi=\frac{1}{3 \ell} \int_{\partial \Omega} r^{3}\left(\cos \varphi+\theta \sin \varphi-\frac{1}{2} \theta^{2} \cos \varphi+\ldots\right) d \varphi
$$

and observes that

$$
\frac{1}{3 \ell} \int_{\partial \Omega} r^{3} \cos \varphi d \varphi=\frac{1}{\ell} \int_{\Omega} x d A=\frac{A \bar{x}}{\ell},
$$

where $\bar{x}$ is the $x$-coordinate of the centroid. Prytz and Hill thus have the approximation

$$
\ell \sigma=\ell^{2} \Delta \theta \approx A+\frac{A \bar{x}}{\ell}+\frac{I_{B}}{2 \ell^{2}}=A\left(1+\frac{\bar{x}}{\ell}+\frac{R_{B}^{2}}{2 \ell^{2}}\right),
$$


where $R_{B}^{2}$ is the mean-square distance of points in $\Omega$ from $B$. Although they don't say it explicitly, it is easily seen that the error in this approximation is $\mathcal{O}\left((d / \ell)^{3}\right)$, where $d$ is the diameter of the set $\Omega \cup\{B\}$ (or simply the diameter of $\Omega$ if $B \in \bar{\Omega}$ ). Thus the error made by the approximation $A \approx \ell \sigma$ is $\mathcal{O}(d / \ell)$.

Note that when measuring small regions, $\Delta \theta$ is small but non-zero, that is, the chisel edge does not come to rest in the place where it started. As we will see in the next section and at the end of the last section, this can happen if the planimeter traces the boundary of a large region or a curve that bounds an oriented area of 0 .

Prytz and Hill recommend measuring the region twice with the same base point but with opposite initial directions, and averaging the results. Prytz's reasoning on this is not clear, but Hill notes that the $\bar{x}$ term will have opposite signs in the two measurements, and so will drop out in the average, giving the approximation

$$
\ell \bar{\sigma} \approx A+\frac{I_{B}}{2 \ell^{2}}=A\left(1+\frac{R_{B}^{2}}{2 \ell^{2}}\right),
$$

where $\bar{\sigma}$ is the average of the displacements in the two measurements. From this, the error in the approximation $A \approx \ell \bar{\sigma}$ is $\mathcal{O}\left((d / \ell)^{2}\right)$. Note that $\bar{x}$ also vanishes if the centroid is on the line through $B$ perpendicular to the initial position of the planimeter, agreeing with the intuitive observation in Section 1 that a good starting position is one that is perpendicular to a line bisecting the region.

The term $I_{B}=A R_{B}^{2}$ is minimized when $B$ is the centroid, which accounts for the recommendation of Prytz and Hill that the tracing start and end at the centroid.

From this analysis it appears that the holonomy $\Delta \theta$ of the Prytz planimeter is some combination of all of the moments of $\Omega$ about the base point $B$. It is hoped that the modern approach taken in the next sections will lead to a better understanding of the relationship between these.

\section{Motion of the Prytz Planimeter as}

Parallel Translation in a Fiber Bundle

The configuration space for the Prytz planimeter is $E=\mathbb{R}^{2} \times S^{1}$, where the first factor is the location of the tracer point and the second factor is the angle giving the direction of the planimeter. We will consider $E \stackrel{\pi}{\longrightarrow} \mathbb{R}^{2}$ as a trivial circle bundle, where $\pi$ is projection onto the first factor. If the tracer point follows a curve $\gamma$ in $\mathbb{R}^{2}$, the resulting motion of the planimeter defines a section of this bundle along $\gamma$. We will see that this motion is described as parallel translation of an Ehresmann connection on $E \stackrel{\pi}{\longrightarrow} \mathbb{R}^{2}$. Thus, when the planimeter traverses the boundary of some region, its net rotation $\Delta \theta$ is an example of the holonomy of this connection. Our main source for connections and parallel translation in fiber bundles is $[\mathrm{KMS}]$, but also see [Poo]. Many of the results in this and the next section illustrate the general theory in [KMS]. 
Intuitively, the motion of the tracer point along a curve induces a one-parameter family of diffeomorphisms of the fiber $S^{1}$ (in which the fibers over different points are identified by projection $E \rightarrow S^{1}$ onto the second factor, i.e., by the triviality of the bundle). It is clear that these diffeomorphisms are not simply rotations. For example, if the tracer point is moved along a straight line, the diffeomorphisms have two common antipodal fixed points, one attracting and one repelling, the repelling fixed point being the fiber element in the direction of motion. As another example, consider the holonomy determined by tracing the boundary of a region that is small relative to the length of the planimeter. As seen in Sections 1 and 3, the resulting diffeomorphism of the initial circle (which is the fiber over the base point where the tracing begins and ends) is only approximately a rotation, since the net rotation of the planimeter depends on its initial direction. Thus $E \stackrel{\pi}{\longrightarrow} \mathbb{R}^{2}$ should not be viewed as a principal bundle in this context, since the group $G$ acting on the fiber is not the group of rotations (more precisely, the connection on $E \stackrel{\pi}{\longrightarrow} \mathbb{R}^{2}$, defined below, is not principal). In the next section we will determine the group $G$, and see that $E \stackrel{\pi}{\longrightarrow} \mathbb{R}^{2}$ is, in fact, an associated bundle of a $G$-principal bundle, and that the connection on $E \stackrel{\pi}{\longrightarrow} \mathbb{R}^{2}$ is induced by a connection on this principal bundle.

Take $(x, y, \theta)$ as coordinates on $E$, where $(x, y)$ are base coordinates and $\theta$ is the fiber coordinate. The tracer point and chisel edge then have coordinates $q=(x, y)$ and $p=$ $(x+\ell \cos \theta, y+\ell \sin \theta)$, respectively, and the forward-pointing normal (from Section 1) is $N=\sin \theta \frac{\partial}{\partial x}-\cos \theta \frac{\partial}{\partial y}$.

The expression $d \sigma=N \cdot d p$, defined in Section 1, is a 1-form on $E$. It is not exact, and henceforth will be denoted simply as $\sigma$. The coordinate expression for $\sigma$ is

$$
\sigma=\sin \theta d x-\cos \theta d y-\ell d \theta
$$

and so the motion of the planimeter is governed by the non-holonomic condition

$$
\sigma=0, \quad \text { or } \quad \ell d \theta=\sin \theta d x-\cos \theta d y
$$

For $e=(q, \theta) \in E$, let $H_{e}=\operatorname{ker} \sigma_{e} \subset T_{e} E$. The vector fields

$$
X=\frac{\partial}{\partial x}+\frac{1}{\ell} \sin \theta \frac{\partial}{\partial \theta} \quad \text { and } \quad Y=\frac{\partial}{\partial y}-\frac{1}{\ell} \cos \theta \frac{\partial}{\partial \theta}
$$

form a basis for $H_{e}$ at each $e \in E$, and $\left.\pi_{*}\right|_{H_{e}}: H_{e} \rightarrow T_{\pi(e)} \mathbb{R}^{2}$ is clearly an isomorphism, where $\pi_{*}$ is the differential of $\pi$. It follows that $H=\left\{H_{e} \mid e \in E\right\}$, viewed as a sub-bundle of $T E \rightarrow E$, forms the distribution of horizontal subspaces of a connection on $E \stackrel{\pi}{\longrightarrow} \mathbb{R}^{2}$ (see [KMS, §9.3]). The connection form is the 1-form $\Phi=-\frac{1}{\ell} \sigma \otimes \frac{\partial}{\partial \theta}$, which takes values in the bundle $V$ of vertical vectors, where $V_{e}=\operatorname{ker}\left(\pi_{*}\right)_{e} \subset T_{e} E$. More precisely, $\Phi_{e}: T_{e} E \rightarrow V_{e}$, defined by $\Phi_{e}(W)=-\left.\frac{1}{\ell} \sigma(W) \frac{\partial}{\partial \theta}\right|_{e}$, is projection onto $V_{e}$ with kernel $H_{e}$. If the tracer 
point of the planimeter follows a piecewise smooth curve $\gamma$ in $\mathbb{R}^{2}$, the resulting motion of the planimeter defines a curve $\tilde{\gamma}$ in $E$ covering $\gamma$ such that $\tilde{\gamma}^{\prime}(t) \in H_{\tilde{\gamma}(t)}$ for all $t$, by virtue of (3). Thus the motion of the planimeter is parallel translation for this connection.

The connection is also determined by its Christoffel form $\Gamma$, which is a 1-form on $\mathbb{R}^{2}$ with values in $\mathfrak{X}\left(S^{1}\right)$, the Lie algebra of smooth vector fields on $S^{1}$. (See [KMS, $\S 9.7$. There needs to be just one Christoffel form since the bundle is trivial.) Suppose the tracer point of the planimeter is at $q \in \mathbb{R}^{2}$ moving with velocity $v=a \frac{\partial}{\partial x}+b \frac{\partial}{\partial y}$. The induced vector field $w=\Gamma(v)=c(\theta) \frac{\partial}{\partial \theta}$ on $S^{1}$ is given by applying (3):

$$
\ell c(\theta)=\ell d \theta\left(w_{\theta}\right)=(\sin \theta d x-\cos \theta d y)(v)=a \sin \theta-b \cos \theta .
$$

Consequently,

$$
\Gamma(v)_{\theta}=w_{\theta}=\frac{1}{\ell}(a \sin \theta-b \cos \theta) \frac{\partial}{\partial \theta} .
$$

More directly, given $\theta \in S^{1}$, let $v_{\theta}=v+0 \frac{\partial}{\partial \theta} \in T_{(q, \theta)} E$. Then $w=\Gamma(v)$ is defined by $\Gamma(v)_{\theta}=-\Phi\left(v_{\theta}\right)=\frac{1}{\ell} \sigma\left(v_{\theta}\right) \frac{\partial}{\partial \theta}$ (an identification is being made here between the fiber $E_{q}=$ $\{q\} \times S^{1}$ and the model fiber $\left.S^{1}\right)$. In either case we can write $\Gamma=\frac{1}{\ell}(\sin \theta d x-\cos \theta d y) \otimes \frac{\partial}{\partial \theta}$.

In general, a connection on a fiber bundle need not be complete, that is, parallel translation need not be defined for all elements of the bundle along all piecewise smooth curves. However if the fiber is compact, as in the present case, then the connection is necessarily complete, and so is properly called an Ehresmann connection. This follows from a comment in [KMS, §9.9], or by the following standard argument. Suppose $\gamma:[0,1] \rightarrow \mathbb{R}^{2}$ is a piecewise smooth curve. Let $\epsilon>0$ and extend $\gamma$ to have domain $I=(-2 \epsilon, 1+2 \epsilon)$, and consider the pull-back connection on the pull-back bundle $\gamma^{*} E \rightarrow I$. Let $f: I \rightarrow \mathbb{R}$ be a function with support in $(-\epsilon, 1+\epsilon)$ that is identically 1 on $[0,1]$. Let $T$ be the horizontal lift of the vector field $f \frac{d}{d t}$ to $\gamma^{*} E$. Then $T$ has compact support (by the compactness of the fiber), and so is a complete vector field. As the flow of $T$ over $[0,1]$ represents parallel translation along $\gamma$ from $\gamma(0)$ to $\gamma(1)$, the connection is complete. Note that this implies the intuitive observation made at the beginning of this section that the motion of the tracer point along a curve induces a one-parameter family of diffeomorphisms of $S^{1}$, or equivalently, a family of diffeomorphisms between the fibers of $E$ over points along the curve.

The vector fields $X$ and $Y$, given in (4), are, respectively, the horizontal lifts of $\frac{\partial}{\partial x}$ and $\frac{\partial}{\partial y}$ for this connection. The curvature is given by the Lie bracket $R(X, Y)=[X, Y]=\frac{1}{\ell^{2}} \frac{\partial}{\partial \theta}$ (see [KMS, §9.4]). The vector fields $X, Y, \frac{\partial}{\partial \theta}$ form a frame on $E$. The dual frame is easily seen to be $d x, d y,-\frac{1}{\ell} \sigma$. In terms of this frame we have $R=\frac{1}{\ell^{2}}(d x \wedge d y) \otimes \frac{\partial}{\partial \theta}$. We see that if the tracer point moves counterclockwise around an infinitesimal rectangle with edges $\frac{\partial}{\partial x}=\pi_{*}(X)$ and $\frac{\partial}{\partial y}=\pi_{*}(Y)$, then the infinitesimal motion of the fiber is $\frac{1}{\ell^{2}} \frac{\partial}{\partial \theta}$, which is also counterclockwise. It follows that the holonomy group (which will be determined in 
the next section) must contain the rigid rotations, although from the results of Sections 1 and 3 , it contains more than this.

Although the connection defined here on $E \rightarrow \mathbb{R}^{2}$ is not principal, it's interesting to observe that the curvature can be computed using a "covariant exterior derivative" as on a principal $S^{1}$-bundle [KMS, $\left.\S 11.5\right]$. If $\alpha$ is a 1 -form on $E$, then $d_{h} \alpha$ is defined to be the horizontal component of $d \alpha$, that is, $d_{h} \alpha(X, Y)=d \alpha(P X, P Y)$ for $X, Y \in T_{e} E$, where $P: T_{e} E \rightarrow T_{e} E$ is projection onto $H_{e}$ with kernel $V_{e}$. Let $\omega=-\frac{1}{\ell} \sigma$ so that $\Phi=\omega \otimes \frac{\partial}{\partial \theta}$. One computes that $d \omega=-\frac{1}{\ell} \omega \wedge(\cos \theta d x+\sin \theta d y)-\frac{1}{\ell^{2}} d x \wedge d y$. The horizontal component of this is $\Omega=d_{h} \omega=-\frac{1}{\ell^{2}} d x \wedge d y$. The curvature form $\Omega$ is related to the curvature tensor $R$ in the usual way, namely, $R=-\Omega \otimes \frac{\partial}{\partial \theta}$.

\section{5. $E \stackrel{\pi}{\longrightarrow} \mathbb{R}^{2}$ AS AN Associated Bundle}

We will now see that the bundle $E=\mathbb{R}^{2} \times S^{1} \stackrel{\pi}{\longrightarrow} \mathbb{R}^{2}$ is an associated bundle of a principal bundle, and that the connection on $E$ is induced from a connection on this principal bundle. As a comparison, and to further Bishop's analogy of the motion of the planimeter with that of a car, see [Fe]. Our main references for principal bundles are [KMS, $\mathrm{KN}]$.

As noted in the previous section, since the connection on $E \rightarrow \mathbb{R}^{2}$ is complete, motion of the tracer point along a piecewise $C^{1}$ curve in $\mathbb{R}^{2}$ induces a one-parameter family of diffeomorphisms. The diffeomorphisms induced by all piecewise $C^{1}$ curves lie in some smallest subgroup of $\operatorname{Diff}\left(S^{1}\right)$, the group of all diffeomorphisms of $S^{1}$. Our first task is to determine this subgroup.

Theorem 1. The diffeomorphisms of $S^{1}$ induced by moving the tracer point of the planimeter along arbitrary piecewise $C^{1}$ curves in $\mathbb{R}^{2}$ form a group, namely, the group $\mathcal{M}_{0}\left(S^{1}\right)$ of Möbius transformations that preserve $S^{1}$ and its orientation.

The proof consists of a number of steps. In the proof and the rest of the paper we will write elements of $S^{1}$ as $e^{i \theta}$ and identify $\mathbb{R}^{2}$ with $\mathbb{C}$ when convenient.

First we show that the collection of diffeomorphisms is a group. The only question is whether the collection is closed under composition. If $\gamma:[0,1] \rightarrow \mathbb{R}^{2}$ is a piecewise $C^{1}$ curve, let $\psi_{\gamma}$ denote the diffeomorphism of $S^{1}$ induced by moving the tracer point along $\gamma$ from $\gamma(0)$ to $\gamma(1)$. Since the connection on $E \rightarrow \mathbb{R}^{2}$ is invariant under translations of $\mathbb{R}^{2}$, then $\psi_{\gamma+v}=\psi_{\gamma}$ for every $v \in \mathbb{R}^{2}$. If $\gamma_{1}, \gamma_{2}:[0,1] \rightarrow \mathbb{R}^{2}$ are two such curves, then $\psi_{\gamma_{2}} \circ \psi_{\gamma_{1}}$ is the diffeomorphism induced by moving the tracer point along the curve $\gamma$ defined by $\gamma(t)=\gamma_{1}(2 t)$ for $t \in\left[0, \frac{1}{2}\right]$ and $\gamma(t)=\gamma_{2}(2 t-1)+\gamma_{1}(1)-\gamma_{2}(0)$ for $t \in\left[\frac{1}{2}, 1\right]$. Thus the collection of diffeomorphisms is closed under composition, and hence is a group.

Before continuing with the proof of Theorem 1, we note some facts related to $\mathcal{M}_{0}\left(S^{1}\right)$.

(1) $\mathcal{M}_{0}\left(S^{1}\right)$ is the same as the group of Möbius transformations that preserve the unit disk $D$, which is, of course, the group of orientation-preserving isometries of the 
Poincare disk model of the hyperbolic plane. These Möbius transformations can be written as $z \mapsto \frac{a z+b}{\bar{b} z+\bar{a}}$. This is a standard fact from elementary complex analysis, of course, but is done particularly nicely in [Se].

(2) The "mixed signature" special unitary group $S U(1,1)=\left\{\left(\frac{a}{b} \frac{b}{a}\right):|a|^{2}-|b|^{2}=1\right\}$ is a double cover of $\mathcal{M}_{0}\left(S^{1}\right)$, the homomorphism being the usual map taking $A=\left(\frac{a}{b} \frac{b}{a}\right)$ to the transformation $A \cdot z=\frac{a z+b}{\bar{b} z+\bar{a}}$. We will use this to identify $\mathcal{M}_{0}\left(S^{1}\right)$ with the projective group $P S U(1,1)=S U(1,1) /\{ \pm I\}$, and consequently to identify the Lie algebra of $\mathcal{M}_{0}\left(S^{1}\right)$ with $\mathfrak{s u}(1,1)=\left\{\left(\begin{array}{cc}i \gamma & \beta \\ \beta & -i \gamma\end{array}\right): \gamma \in \mathbb{R}, \beta \in \mathbb{C}\right\}$. This is an easy exercise, and is done implicitly in [Se]. It is completely analogous to the more common identification of $S L(2, \mathbb{R})$ as a double cover of the Möbius transformations preserving the upper half-plane (see, e.g., $[\mathrm{Bo}]) . S U(1,1)$ and $S L(2, \mathbb{R})$ are conjugate subgroups of $S L(2, \mathbb{C})$, related by any matrix representing a Möbius transformation mapping $D$ onto the upper half-plane.

(3) By restricting to $S^{1}, \mathcal{M}_{0}\left(S^{1}\right)$ injects into $\operatorname{Diff}\left(S^{1}\right)$. The differential of this is a Lie algebra injection $\varphi: \mathfrak{s u}(1,1) \rightarrow \mathfrak{X}\left(S^{1}\right)$. More precisely, let $X \in \mathfrak{s u}(1,1)$. To compute the vector field $\varphi(X)$ at $z_{0}=e^{i \theta_{0}} \in S^{1}$, let $e^{i \theta(t)}=e^{t X} \cdot z_{0}$. Then

$$
\varphi(X)_{z_{0}}=\left.\theta^{\prime}(0) \frac{\partial}{\partial \theta}\right|_{z_{0}}=\left.\left(-\left.i \bar{z}_{0} \frac{d}{d t}\right|_{0} e^{t X} \cdot z_{0}\right) \frac{\partial}{\partial \theta}\right|_{z_{0}} .
$$

Next we determine how the Christoffel form $\Gamma: T \mathbb{R}^{2} \rightarrow \mathfrak{X}\left(S^{1}\right)$ and the map $\varphi: \mathfrak{s u}(1,1) \rightarrow$ $\mathfrak{X}\left(S^{1}\right)$ are related.

Lemma 2. The Christoffel form $\Gamma$ takes values in the image of $\varphi$. In particular, if $v=$ $a \frac{\partial}{\partial x}+b \frac{\partial}{\partial y}$, then $\Gamma(v)=\varphi(X)$, where $X=-\frac{1}{2 \ell}\left(\begin{array}{cc}0 & a+i b \\ a-i b & 0\end{array}\right)$.

Proof. Suppose the tracer point is at $q \in \mathbb{R}^{2}$ moving with velocity $v=a \frac{\partial}{\partial x}+b \frac{\partial}{\partial y}$. From (5), the induced vector field on $S^{1}$ is

$$
\Gamma(v)_{z_{0}}=\left.\frac{1}{\ell}\left(a \sin \theta_{0}-b \cos \theta_{0}\right) \frac{\partial}{\partial \theta}\right|_{z_{0}}
$$

where $z_{0}=e^{i \theta_{0}}$. We need to identify $\Gamma(v)$ as $\varphi(X)$ for some $X \in \mathfrak{s u}(1,1)$.

Let $X=\left(\begin{array}{cc}0 & \beta \\ \beta & 0\end{array}\right)$, and let $\left(\begin{array}{cc}a(t) & b(t) \\ \bar{b}(t) & \bar{a}(t)\end{array}\right)=e^{t X}$. From $(6)$ we get $\varphi(X)_{z_{0}}=\left.2 \operatorname{Im}\left(\beta \bar{z}_{0}\right) \frac{\partial}{\partial \theta}\right|_{z_{0}}$. If $\beta=a+b i$, then $\varphi(X)_{z_{0}}=\left.2\left(b \cos \theta_{0}-a \sin \theta_{0}\right) \frac{\partial}{\partial \theta}\right|_{z_{0}}$. Comparing this with (7), it follows that $\Gamma(v)=\varphi\left(-\frac{1}{2 \ell} X\right)$.

Let $\gamma:[0,1] \rightarrow \mathbb{R}^{2}$ be a $C^{1}$ curve. We seek a curve in $\mathcal{M}_{0}\left(S^{1}\right)$ (actually its lift in $S U(1,1)$ ) that generates the same one-parameter family of diffeomorphisms as $\gamma$. Suppose 
$A:[0,1] \rightarrow S U(1,1)$ is a $C^{1}$ curve, and consider the resulting flow on $S^{1}$. At $t=t_{0}$ the vector field for the flow is given (in a computation similar to that in (6)) by

$$
\left.\left(-\left.i \bar{z}_{0} \frac{d}{d t}\right|_{t_{0}} A(t) A\left(t_{0}\right)^{-1} \cdot z_{0}\right) \frac{\partial}{\partial \theta}\right|_{z_{0}}=\varphi\left(A^{\prime}\left(t_{0}\right) A\left(t_{0}\right)^{-1}\right)_{z_{0}} .
$$

For $A$ and $\gamma$ to generate the same vector field on $S^{1}$ at time $t$, we must have $\Gamma\left(\gamma^{\prime}(t)\right)=$ $\varphi\left(A^{\prime}(t) A(t)^{-1}\right)$. Define the $\mathfrak{s u}(1,1)$-valued 1 -form $\omega$ on $\mathbb{R}^{2}$ by $\omega(v)=-\varphi^{-1}(\Gamma(v))$, that is, $\omega(v)$ is the matrix in $\mathfrak{s u}(1,1)$ that gives rise to the vector field $-\Gamma(v)$ on $S^{1}$. From the lemma, we see that the formula for $\omega$ is $\omega=\frac{1}{2 \ell}\left(\begin{array}{cc}0 & d z \\ d \bar{z} & 0\end{array}\right)$. Then the desired curve in $S U(1,1)$ is the solution of the initial value problem

$$
A^{\prime}(t) A(t)^{-1}=-\omega\left(\gamma^{\prime}(t)\right), \quad A(0)=I
$$

It follows that the group of diffeomorphisms of $S^{1}$ generated by the motion of the tracer point is a subgroup of $\mathcal{M}_{0}\left(S^{1}\right)$. By the lemma, the values of $\omega$ are of the form $\left(\begin{array}{c}0 \\ \beta\end{array}\right)$. These generate the entire Lie algebra $\mathfrak{s u}(1,1)$, and so it follows that the group is $\mathcal{M}_{0}\left(S^{1}\right)$. This completes the proof of Theorem 1.

From the proof of the theorem we see that the action of the one-parameter subgroup $e^{-t \omega(v)}$ on $S^{1}$ is the same as that of moving the tracer point in a straight line in $\mathbb{R}^{2}$ with constant velocity $v$. If the tracer point starts at $q \in \mathbb{R}^{2}$ with the planimeter in the direction $e^{i \theta_{0}}$, then the resulting curve in $E=\mathbb{R}^{2} \times S^{1}$ is $\left(q+t v, e^{-t \omega(v)} \cdot e^{i \theta_{0}}\right)$. Identifying $\mathbb{C}$ and $\mathbb{R}^{2}$, the path of the chisel, which is a standard tractrix, is $\tau(t)=(q+t v)+\ell e^{-t \omega(v)} \cdot e^{i \theta_{0}}$. Letting $q=0$ and $v=\frac{\partial}{\partial x}$ (identified with $1=1+0 i$ in $q+t v$ ), we have $e^{-t \omega(v)}=$ $\left(\begin{array}{cc}\cosh (t / 2 \ell) & -\sinh (t / 2 \ell) \\ -\sinh (t / 2 \ell) & \cosh (t / 2 \ell)\end{array}\right)$ and

$$
\begin{aligned}
\tau(t) & =t+\frac{\cosh (t / 2 \ell) e^{i \theta_{0}}-\sinh (t / 2 \ell)}{-\sinh (t / 2 \ell) e^{i \theta_{0}}+\cosh (t / 2 \ell)} \\
& =t+\ell \frac{\cosh (t / \ell) \cos \theta_{0}-\sinh (t / \ell)}{\cosh (t / \ell)-\sinh (t / \ell) \cos \theta_{0}}+i \ell \frac{\sin \theta_{0}}{\cosh (t / \ell)-\sinh (t / \ell) \cos \theta_{0}}
\end{aligned}
$$

When $\theta_{0}=\pi / 2$, this simplifies to $\tau(t)=t-\ell \tanh (t / \ell)+i \ell \operatorname{sech}(t / \ell)$, which is $(2)$ in complex form.

Now consider $S^{1}$ as the points at infinity of the Poincaré disk model $D$ of the hyperbolic plane. Acting on $D, e^{-t \omega(v)}$ is the one-parameter group of hyperbolic translations that moves the origin along a geodesic with initial velocity $-\frac{v}{2 \ell}$ (note that this is in the opposite direction of the motion of the tracer point). This has the following nice interpretation. Suppose that a hyperbolic stargazer goes walking with constant velocity $v$ in $D$. It is 
natural for the stargazer to think of herself as always being at the center of the celestial circle, with the hyperbolic plane passing beneath her feet with constant velocity $-v$. If she fixes her gaze on a particular star, then the retrograde motion of that star is the same as the motion of a (Euclidean!) Prytz planimeter of length $\ell=1 / 2$.

This discussion shows how a geodesic in $\mathbb{R}^{2}$ can be developed into a geodesic in $D$. Generalizing this, one can develop any polygonal path in $\mathbb{R}^{2}$ to a polygonal path in $D$ (and by polygonal approximations, any piecewise $C^{1}$ path). One needs to be careful, however. A sequence $e^{X_{1}}, \ldots, e^{X_{n}}$ of translations that moves the origin around a closed path in $D$ determines a rotation (relative to the origin) of the points at infinity, whereas the tracer point of the planimeter following a closed loop typically does not result in a pure rotation of the initial directions of the planimeter. Evidently, the motion of the tracer point that induces the same sequence $e^{X_{1}}, \ldots, e^{X_{n}}$ is not generally a closed path in $\mathbb{R}^{2}$. Similarly, the motion of the tracer point in $\mathbb{R}^{2}$ around a closed path does not generally develop to a closed path in $D$.

As a consequence of Theorem 1 , we view $E \rightarrow \mathbb{R}^{2}$ as an $\mathcal{M}_{0}\left(S^{1}\right)$-bundle or as an $S U(1,1)$-bundle [KMS, $§ 10.1]$. The form $\omega$ in the proof is, of course, a "local frame representation" of a connection on some principal bundle [KN, pg. 66] (a "physicist's connection" in [KMS, §11.4]), and (8) is the corresponding parallel translation equation. Writing down the principal bundle and realizing $E \rightarrow \mathbb{R}^{2}$ as an associated bundle is now straight forward.

For the remainder of this section, let $G$ be $\mathcal{M}_{0}\left(S^{1}\right)$ or $S U(1,1)$. Consider the principal $G$-bundle $P=\mathbb{R}^{2} \times G \rightarrow \mathbb{R}^{2}$. As $G$ acts on $S^{1}$, we have the standard construction of the associated $S^{1}$ fiber bundle $\tilde{E} \rightarrow \mathbb{R}^{2}$ (see [KN, pg. 54; KMS, §10.7]). The space $\tilde{E}$ is given by $\left(P \times S^{1}\right) / \sim$, in which $\left(q, A, e^{i \theta}\right) \sim\left(q, I, A \cdot e^{i \theta}\right)$. The map $E \rightarrow \tilde{E}$ that takes $\left(q, e^{i \theta}\right)$ to the equivalence class of $\left(q, I, e^{i \theta}\right)$ is clearly a fiber bundle equivalence.

Each element $(q, A)$ in the fiber $\{q\} \times G$ of $P \rightarrow \mathbb{R}^{2}$ represents a "frame" for the fiber $\{q\} \times S^{1}$ of $E \rightarrow \mathbb{R}^{2}$, that is, a diffeomorphism from the model fiber $S^{1}$ to $\{q\} \times S^{1}$ given by $e^{i \theta} \mapsto\left(q, A \cdot e^{i \theta}\right)$. As both bundles are trivial, it is easiest to make computations relative to the "standard frame," that is, the "identity" diffeomorphism given by projection $\{q\} \times S^{1} \rightarrow S^{1}$, represented by $(q, I) \in P$. The standard frame is more than a computational convenience, however, since it represents the Euclidean geometry of the plane. The identification it makes of the fibers of $E \rightarrow \mathbb{R}^{2}$ is by Euclidean translation. Writing the connections on $E \rightarrow \mathbb{R}^{2}$ and $P \rightarrow \mathbb{R}^{2}$ using the "local descriptions" of $\Gamma$ and $\omega$ amounts to describing how the motion of the planimeter differs from Euclidean parallel translation of vectors.

The connection form $\tilde{\omega}: T P \rightarrow \mathfrak{s u}(1,1)$ along the identity section of $P \rightarrow \mathbb{R}^{2}$ is given as follows. If $v \oplus X \in T_{(q, I)} P=T_{q} \mathbb{R}^{2} \oplus \mathfrak{s u}(1,1)$, then $\tilde{\omega}(v \oplus X)=\omega(v)+X$. Note that $\tilde{\omega}(v \oplus X)=0$ implies $X=-\omega(v)$, that is, $v \oplus X$ is horizontal when $X$ induces the same vector field on $S^{1}$ as the planimeter when the tracer point undergoes the infinitesimal 
displacement $v$. The connection form $\tilde{\omega}$ is extended off of the identity section by the usual equivariance requirement [KN, pg. 64; KMS, §11.1]: if $v \oplus X A \in T_{(q, A)} P$, then $\tilde{\omega}(v \oplus X A)=$ $\operatorname{Ad}\left(A^{-1}\right)(\tilde{\omega}(v \oplus X))=\operatorname{Ad}\left(A^{-1}\right)(\omega(v)+X)$. It is clear from the proof of Theorem 1 that the induced connection on $E \rightarrow \mathbb{R}^{2}$ viewed as an associated bundle is the same as the connection $-\frac{1}{\ell} \sigma \otimes \frac{\partial}{\partial \theta}$ in the previous section, as they induce the same parallel translation. This also follows from Lemma 2 and the theorem in [KMS, §11.9].

Finally, we compute the curvature and holonomy. Relative to the standard frame, the curvature form is

$$
\Omega=d \omega+\omega \wedge \omega=\frac{1}{4 \ell^{2}}\left(\begin{array}{cc}
d z \wedge d \bar{z} & 0 \\
0 & d \bar{z} \wedge d z
\end{array}\right)=-\frac{1}{\ell^{2}}\left(\begin{array}{cc}
i / 2 & 0 \\
0 & -i / 2
\end{array}\right) d x \wedge d y
$$

The infinitesimal rotations in $\mathfrak{s u}(1,1)$ are of the form $\left(\begin{array}{cc}i \gamma & 0 \\ 0 & -i \gamma\end{array}\right)$. Thus the curvature is purely rotational, at least relative to the standard frame. If $Z=\left(\begin{array}{cc}i \gamma & 0 \\ 0 & -i \gamma\end{array}\right)$, then $e^{Z}=$ $\left(\begin{array}{cc}e^{i \gamma} & 0 \\ 0 & e^{-i \gamma}\end{array}\right)$, which acts as a rotation through angle $2 \gamma$. Remembering that $-\Omega$ should be a measure of infinitesimal holonomy, let $Z=-\Omega\left(\frac{\partial}{\partial x}, \frac{\partial}{\partial y}\right)=\frac{1}{\ell^{2}}\left(\begin{array}{cc}i / 2 & 0 \\ 0 & -i / 2\end{array}\right)$. Then $e^{t Z}$ induces the infinitesimal rotation $\varphi(Z)=\frac{1}{l^{2}} \frac{\partial}{\partial \theta}$, agreeing with the bracket curvature computation $R(X, Y)=[X, Y]$ from the previous section, where $X$ and $Y$ are the horizontal lifts in $E$ of $\frac{\partial}{\partial x}$ and $\frac{\partial}{\partial y}$ given in (4).

For $p \in \mathbb{R}^{2}$ let $\mathcal{H}_{p} \subset G$ be the holonomy group based at $p$ for the connection on $P \rightarrow \mathbb{R}^{2}$, and let $\mathfrak{h}_{p}$ be its Lie algebra. The curvature computation shows that $\mathfrak{h}_{p}$ contains the infinitesimal rotations of $E_{p}=\{p\} \times S^{1}$, and so $\mathcal{H}_{p}$ contains the rotations of $E_{p}$ (or their representations in $S U(1,1)$ ), even though we have not yet seen a closed curve that induces a rotation as its holonomy. By the Ambrose-Singer Theorem [KN, pg. 89], if $Z=\Omega\left(\left.\frac{\partial}{\partial x}\right|_{q},\left.\frac{\partial}{\partial y}\right|_{q}\right)$ is an infinitesimal rotation at some other point $q$, then $\psi_{\gamma}^{-1} Z \psi_{\gamma}$ is in $\mathfrak{h}_{p}$, where $\psi_{\gamma}$ is parallel translation for the connection in $P \rightarrow \mathbb{R}^{2}$ along some curve $\gamma$ from $p$ to $q$. In particular, we can parallel translate along the segment joining $p$ and $q$. Let $v=q-p$. Then the proof of Theorem 1 (particularly Lemma 2), shows that parallel translation from $p$ to $q$ is represented by $e^{X}$ in $G$, where $X=-\omega(v)$. Thus $e^{-X} Z e^{X}$ is in $\mathfrak{h}_{p}$; it is the infinitesimal holonomy resulting from moving the tracer point along the segment from $p$ to $q$, around a small loop, and back along the segment to $p$. It follows that $\left.\frac{d}{d t}\right|_{0}\left(e^{-t X} Z e^{t X}\right)=-[X, Z]$ is in $\mathfrak{h}_{p}$. As we have seen, $X$ has the form $\left(\begin{array}{cc}0 & \beta \\ \beta & 0\end{array}\right)$. But $-[X, Z]=2\left(\begin{array}{cc}0 & i \beta \\ -i \bar{\beta} & 0\end{array}\right)$ has the same form, and these generate the Lie algebra $\mathfrak{s u}(1,1)$, as noted earlier. It follows that $\mathcal{H}_{p}$ is the entire group $G$. When $\mathcal{H}_{p}$ acts on $E_{p}$, it follows that the holonomy group at $p$ for the bundle $E \rightarrow \mathbb{R}^{2}$ is $\mathcal{M}_{0}\left(S^{1}\right)$. 
The holonomy for $E \rightarrow \mathbb{R}^{2}$ could also be determined by computing the Lie algebra generated by the vector fields $\Gamma(v)$, and appealing to the generalized Ambrose-Singer Theorem due to Michnor [KMS, §9.11]. An easy computation shows that this Lie algebra is the image of the map $\varphi: \mathfrak{s u}(1,1) \rightarrow \mathfrak{X}\left(S^{1}\right)$ used in Lemma 2 , which is isomorphic to $\mathfrak{s u}(1,1)$.

This is all summarized in the following theorem.

Theorem 3. Let $G$ be $\mathcal{M}_{0}\left(S^{1}\right)$ or $S U(1,1)$.

(1) The bundle $E=\mathbb{R}^{2} \times S^{1} \rightarrow \mathbb{R}^{2}$ is (isomorphic to) an associated bundle of the principal bundle $P=\mathbb{R}^{2} \times G \rightarrow \mathbb{R}^{2}$.

(2) The motion of the planimeter is given by parallel translation for a connection on $E \rightarrow \mathbb{R}^{2}$, which is induced by a principal connection on $P \rightarrow \mathbb{R}^{2}$. With respect to the standard frame, the connection and curvature forms for the principal connection are $\omega=\frac{1}{2 \ell}\left(\begin{array}{cc}0 & d z \\ d \bar{z} & 0\end{array}\right)$ and $\Omega=-\frac{1}{\ell^{2}}\left(\begin{array}{cc}i / 2 & 0 \\ 0 & -i / 2\end{array}\right) d x \wedge d y$.

(3) The holonomy group for the connection on $P \rightarrow \mathbb{R}^{2}$ is $G$.

(4) The holonomy group for the connection on $E \rightarrow \mathbb{R}^{2}$ is $\mathcal{M}_{0}\left(S^{1}\right)$.

Questions and Speculations. The motion of the tracer point around a closed curve, starting and stopping at a base point, determines an element of the holonomy group. If a different base point on the curve is used, the two holonomy elements need not be the same, but are conjugate to each other. (The holonomy groups at the two different base points are identified by the Euclidean translation that identifies the corresponding fibers of $E \rightarrow \mathbb{R}^{2}$.) Thus a region $\Omega$ with a simple, closed, piecewise $C^{1}$ boundary determines a conjugacy class in $G$. What information about $\Omega$ can be determined from its conjugacy class?

Suppose $\Omega$ is sufficiently small relative to the length $\ell$ of the planimeter so that the holonomy $H$ determined by tracing $\partial \Omega$ starting at base point $B$ acts on $S^{1}$ without fixed points. When $H$ acts on the Poincaré disk $D$, it is a hyperbolic rotation about some point $z(B) \in D$ (see the next section). Every hyperbolic rotation is conjugate to a rotation about the origin. In particular, let $\varphi_{B}(\zeta)=\frac{\zeta-z(B)}{1-\bar{z}(B) \zeta}$. Then $\varphi_{B}$ is the hyperbolic translation taking $z(B)$ to the origin, and $\varphi_{B} H \varphi_{B}^{-1}$ is a rotation about the origin. The transformation $\varphi_{B}$ is represented by $\left(\begin{array}{cc}a & b \\ b & a\end{array}\right)$ in $S U(1,1)$, where $a=1 / \sqrt{1-|z(B)|^{2}}$ and $b=-z(B) / \sqrt{1-|z(B)|^{2}}$. From our results above, this matrix is $e^{-\omega(v)}$ for some $v \in \mathbb{R}^{2}$. Letting $f(B)$ be the point $B+v$, it follows that $f(B)$ is the unique point in $\mathbb{R}^{2}$ with the following property. If the planimeter starts tracing at $f(B)$, moves along the segment joining $f(B)$ to $B$, goes around $\partial \Omega$, and then back along the segment to $f(B)$, then the resulting holonomy is purely rotational. In this way the curve $\partial \Omega$ and the number $\ell>0$ determine curves $z: \partial \Omega \rightarrow D$ and $f: \partial \Omega \rightarrow \mathbb{R}^{2}$. Are these curves related to $\partial \Omega$ in some simpler way? 
Given an element of the holonomy group, it would be nice to find a loop, perhaps of shortest length, that generates it. In particular, what curves generate pure rotations in $G$ ? Some are given in the previous paragraph, but can they be characterized more simply? What non-trivial curves generate the identity? When $G=S U(1,1)$, what curves generate holonomy $-I$ ?

For the Prytz planimeter on the plane, it's easiest to refer everything to the standard frame, since the bundles are all trivial. The full machinery of the principal bundle viewpoint may be necessary to study how the planimeter works on the sphere. In this case the configuration space $E \rightarrow S^{2}$ is the unit circle bundle over the sphere, which would appear to be an associated bundle of an appropriate principal $G$-bundle over $S^{2}$, as in the $\mathbb{R}^{2}$ case. The Hopf fibration factors as $S^{3} \rightarrow E \rightarrow S^{2}$ (the first map is a double cover), and so it seems that the connection on $E \rightarrow S^{2}$ given by the motion of the planimeter should lift to a connection on the Hopf fibration, which would have $S U(1,1)$ as its group.

\section{A Special Case of Menzin's Conjecture}

Recall Menzin's conjecture from Section 2: If a planimeter of length $\ell$ traces the boundary of a region $\Omega$ with area $A>\pi \ell^{2}$, then the chisel edge asymptotically approaches a particular tractrix that is closed. When the planimeter follows this closed tractrix, it makes a complete rotation. In this section we prove this in the case when $\partial \Omega$ is a parallelogram.

The conjecture can be rephrased in terms of the Poincaré return map on the initial circle and the winding number of a section of $E \rightarrow \mathbb{R}^{2}$ over $\partial \Omega$. Let $B$ be a base point on $\partial \Omega$, and let $H: S^{1} \rightarrow S^{1}$ be the diffeomorphism in $\mathcal{M}_{0}\left(S^{1}\right)$ defined by tracing $\partial \Omega$ in the positive direction with the planimeter starting and stopping at $B$, that is, $H$ is the holonomy defined by the curve $\partial \Omega$ and the base point $B$. If the planimeter starts with a fixed point of $H$ as its initial direction, then the motion of the planimeter determines a section of $E \rightarrow \mathbb{R}^{2}$ over $\partial \Omega$. Menzin's conjecture then becomes the following.

Menzin's Conjecture. If $A>\pi \ell^{2}$, then $H$ has a unique attracting fixed point. If the planimeter starts with this fixed point as its initial position, then the induced section of $E \rightarrow \mathbb{R}^{2}$ over $\partial \Omega$ has winding number 1 .

Note that for topological reasons, one generically expects to get a repelling fixed point as well. Menzin's conjecture also predicts this - the repelling fixed point is the attracting fixed point when the tracing direction is reversed.

In contrast, if the planimeter traces a region that is small relative to its length, the results of Prytz and Hill imply that $H$ has no fixed points, as observed earlier.

We recall a standard fact about elements of $\mathcal{M}_{0}\left(S^{1}\right)$ (actually their representatives in $S U(1,1))$, their fixed points on $S^{1}$, and how they act as isometries on the Poincaré disk $D$ (see, e.g., $[\mathrm{Se}])$ :

(1) $A \in S U(1,1)$ has two fixed points on $S^{1}$ if and only if $|\operatorname{tr} A|>2$. In this case $A$ is a hyperbolic translation in $D$ along the geodesic joining the fixed points on $S^{1}$. One of the fixed points is attracting and the other is repelling. 
(2) $A$ has one fixed point on $S^{1}$ if and only if $|\operatorname{tr} A|=2$ and $A \neq \pm I$. In this case $A$ is a horocyclic rotation on $D$ (a motion of $D$ that preserves every horocycle through the fixed point). The fixed point is semi-attracting, that is, it is attracting from one side, but repelling from the other.

(3) $A$ has no fixed points on $S^{1}$ if and only if $|\operatorname{tr} A|<2$. In this case $A$ has a fixed point in $D$ and is a hyperbolic rotation about this point.

Evidently any proof of Menzin's conjecture will involve showing that $A>\pi \ell^{2}$ implies $|\operatorname{tr} H|>2$.

The results of Prytz and Hill combined with Menzin's conjecture yield qualitatively different holonomies when tracing the boundaries of small and large regions. The holonomy of the null curve (the curve that stays at the base point) is, of course, $I$, which has trace 2 , and so is on the boundary between the open sets $U_{0}$ and $U_{2}$ in $S U(1,1)$ of transformations with no fixed points and two fixed points. Traversing a small loop will yield a holonomy $H$ close to $I$. If the loop is the boundary of a region, then $H \in U_{0}$. It is possible to traverse a small loop so that $H \in U_{2}$, but the loop will necessarily have to enclose some area in the positive sense and some in the negative sense, as in a figure eight. An example of this will follow the proof of the special case.

Proof of Menzin's conjecture when $\partial \Omega$ is a parallelogram. Let $v$ and $w$ form a positively oriented basis of $\mathbb{R}^{2}$, and let $\partial \Omega$ be the parallelogram with vertices $0, v, v+w, w$. We will compute the holonomy determined by tracing $\partial \Omega$ in the positive direction with 0 as the base point. Let

$$
\begin{aligned}
& X=\left(\begin{array}{cc}
0 & \beta \\
\beta & 0
\end{array}\right)=-\omega(v)=-\frac{1}{2 \ell}\left(\begin{array}{cc}
0 & d z(v) \\
d \bar{z}(v) & 0
\end{array}\right) \\
& \text { and } \quad Y=\left(\begin{array}{ll}
0 & \delta \\
\delta & 0
\end{array}\right)=-\omega(w)=-\frac{1}{2 \ell}\left(\begin{array}{cc}
0 & d z(w) \\
d \bar{z}(w) & 0
\end{array}\right),
\end{aligned}
$$

and note that

$$
A=\operatorname{Im}(\bar{v} w)=4 \ell^{2} \operatorname{Im}(\bar{\beta} \delta)=4 \ell^{2}|\beta \delta| \sin \theta
$$

where $\theta$ is the angle between $v$ and $w$.

We have $e^{X}=\left(\begin{array}{cc}a & b \\ b & a\end{array}\right)=\left(\begin{array}{cc}a & \alpha \beta \\ \alpha \bar{\beta} & a\end{array}\right)$ and $e^{Y}=\left(\begin{array}{cc}c & d \\ \bar{d} & c\end{array}\right)=\left(\begin{array}{cc}c & \gamma \delta \\ \gamma \bar{\delta} & c\end{array}\right)$, where $a=\cosh |\beta|$, $\alpha=\frac{\sinh |\beta|}{|\beta|}, b=\alpha \beta, c=\cosh |\delta|, d=\gamma \delta$, and $\gamma=\frac{\sinh |\delta|}{|\delta|}$.

The holonomy is then $H=e^{-Y} e^{-X} e^{Y} e^{X}$, which can be written as

$$
H=I+\tilde{H}=I+2 \operatorname{Im}(\bar{b} d)\left(\begin{array}{cc}
i & 0 \\
0 & -i
\end{array}\right) e^{Y} e^{X}
$$

Although it's not essential to the proof, it's nice to observe that

$$
\tilde{H} e^{-X} e^{-Y}=2 \operatorname{Im}(\bar{b} d)\left(\begin{array}{cc}
i & 0 \\
0 & -i
\end{array}\right)=\alpha \gamma \frac{A}{\ell^{2}}\left(\begin{array}{cc}
i / 2 & 0 \\
0 & -i / 2
\end{array}\right)=\alpha \gamma[Y, X]
$$


which should be compared with the expressions for infinitesimal holonomy in the previous two sections.

We need to show that $|\operatorname{tr} H|>2$. One easily computes that $\operatorname{tr} H=2-4 \operatorname{Im}^{2}(\bar{b} d)$. Thus $\operatorname{tr} H$ cannot be bigger than 2 , and $\operatorname{tr} H<-2$ if and only if $\operatorname{Im}(\bar{b} d)>1$. It follows that $H$ has an attracting fixed point if and only if

$$
\operatorname{Im}(\bar{b} d)=\alpha \gamma \operatorname{Im}(\bar{\beta} \delta)=\alpha \gamma|\beta \delta| \sin \theta=\alpha \gamma \frac{A}{4 \ell^{2}}>1, \quad \text { i.e., } \quad A>\frac{4}{\alpha \gamma} \ell^{2}
$$

As $\alpha$ and $\gamma$ are larger than 1, this is close to the hypothesis $A>\pi \ell^{2}$ ! Using the expressions for $\alpha$ and $\gamma$, we have that $H$ has an attracting fixed point if and only if $\operatorname{Im}(\bar{b} d)=\sinh |\beta| \sinh |\delta| \sin \theta>1$.

Using (9), the implication

$$
A>\pi \ell^{2} \quad \Longrightarrow \quad H \text { has an attracting fixed point }
$$

becomes

$$
|\beta \delta| \sin \theta>\frac{\pi}{4} \quad \Longrightarrow \quad \sinh |\beta| \sinh |\delta| \sin \theta>1 .
$$

One easily finds that the minimum of $\sinh x \sinh y \sin \theta$ subject to the constraint $x y \sin \theta \geq$ $\pi / 4$ is $(\cosh \sqrt{\pi}-1) / 2$, which is approximately 1.014 , and so this implication holds.

To see that the planimeter makes a full rotation as the chisel edge follows one of the periodic trajectories, we compute the fixed points of $H$ and observe that the planimeter always rotates counterclockwise. If this is to happen, then the planimeter should make half a rotation as the tracer point moves from one vertex of the parallelogram to the opposite vertex. Consequently we look for solutions of $e^{Y} e^{X} \cdot z=-z$, that is,

$$
\frac{(a c+\bar{b} d) z+(a d+b c)}{(a \bar{d}+\bar{b} c) z+(a c+b \bar{d})}=-z
$$

(this equation is considerably easier than $H \cdot z=z$ ). Using $a^{2}-|b|^{2}=c^{2}-|d|^{2}=1$, the discriminant of this quadratic simplifies to $4\left(1-\operatorname{Im}^{2}(\bar{b} d)\right)$, which is negative by (10). The solutions can then be written as

$$
z_{ \pm}=-\frac{a d+b c}{|a d+b c|} \frac{a c+\operatorname{Re}(\bar{b} d) \pm i \sqrt{\operatorname{Im}^{2}(\bar{b} d)-1}}{|a d+b c|}
$$

(this is to be taken as $z_{+}$and $z_{-}$, using the corresponding sign on the radical). It is easily shown that these are indeed the fixed points of $H$. Both fractions in this expression have unit modulus. Since $a^{2} c^{2}=\left(|b|^{2}+1\right)\left(|d|^{2}+1\right)>|\bar{b} d|^{2} \geq \operatorname{Re}^{2}(\bar{b} d)$, we have $a c+\operatorname{Re}(\bar{b} d)>0$, and so the two values of the second fraction form a conjugate pair with positive real part. Thus the fixed points of $H$ consist of this conjugate pair rotated through the angle 
$\arg (-(a d+b c))$. Thought of as vectors in $\mathbb{R}^{2}, b$ and $d$ point in the directions opposite those of the vectors $v$ and $w$ that give the sides of the parallelogram (see (9)). Thus it is plausible that the conjugate pair of the second fraction above are rotated into the interior of the angle formed by $v$ and $w$ (see Figure 9). This is in fact the case, as will now be shown.

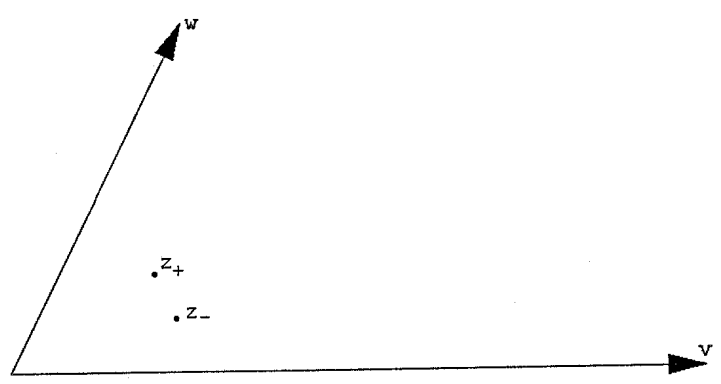

Figure 9.

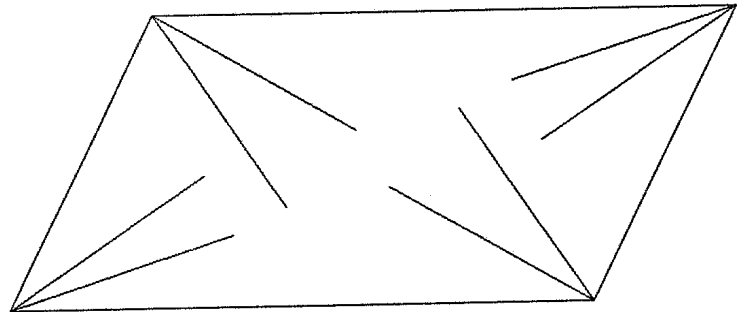

Figure 10.

To be more precise, we need to show that $\arg \left(z_{ \pm} / v\right)=\arg \left(-z_{ \pm} / b\right)$ and $\arg \left(w / z_{ \pm}\right)=$ $\arg \left(-d / z_{ \pm}\right)$are positive, thinking of $v$ and $w$ as complex numbers. The condition $\arg \left(-z_{ \pm} / b\right)>0$ is equivalent to $\operatorname{Im}\left(-z_{ \pm} \bar{b}\right)>0$, which we now show. Proving $\arg \left(-d / z_{ \pm}\right)>$ 0 is similar. We have

$$
|a d+b c|^{2} \operatorname{Im}\left(-z_{ \pm} \bar{b}\right)=a \operatorname{Im}(\bar{b} d)(a c+\operatorname{Re}(\bar{b} d)) \pm\left(a \operatorname{Re}(\bar{b} d)+|b|^{2} c\right) \sqrt{\operatorname{Im}^{2}(\bar{b} d)-1}
$$

For this to be positive, we need

$$
a \operatorname{Im}(\bar{b} d)(a c+\operatorname{Re}(\bar{b} d))>\left.|a \operatorname{Re}(\bar{b} d)+| b\right|^{2} c \mid \sqrt{\operatorname{Im}^{2}(\bar{b} d)-1}
$$

Since $\operatorname{Im}(\bar{b} d)>0$ by (10) and $a c+\operatorname{Re}(\bar{b} d)>0$ (as observed above), this is equivalent to

$$
a^{2} \operatorname{Im}^{2}(\bar{b} d)(a c+\operatorname{Re}(\bar{b} d))^{2}>\left(a^{2} \operatorname{Re}^{2}(\bar{b} d)+2 a|b|^{2} c \operatorname{Re}(\bar{b} d)+|b|^{4} c^{2}\right)\left(\operatorname{Im}^{2}(\bar{b} d)-1\right) .
$$

On the right hand side replace $a^{2}$ with $|b|^{2}+1$, and $|b|^{4}$ with $|b|^{2}\left(a^{2}-1\right)$. Collecting the terms containing $|b|^{2}$ other than $|b|^{2} c^{2}$, we get the equivalent inequality

$$
a^{2} \operatorname{Im}^{2}(\bar{b} d)(a c+\operatorname{Re}(\bar{b} d))^{2}>\left(|b|^{2}(a c+\operatorname{Re}(\bar{b} d))^{2}+\operatorname{Re}^{2}(\bar{b} d)-|b|^{2} c^{2}\right)\left(\operatorname{Im}^{2}(\bar{b} d)-1\right) .
$$

Collecting the terms with $(a c+\operatorname{Re}(\bar{b} d))^{2}$ and using $a^{2}-|b|^{2}=1$, this becomes

$$
\left(|b|^{2}+\operatorname{Im}^{2}(\bar{b} d)\right)(a c+\operatorname{Re}(\bar{b} d))^{2}>\left(\operatorname{Re}^{2}(\bar{b} d)-|b|^{2} c^{2}\right)\left(\operatorname{Im}^{2}(\bar{b} d)-1\right) .
$$

Using $c^{2}=|d|^{2}+1$, we have $\operatorname{Re}^{2}(\bar{b} d)-|b|^{2} c^{2}=-\left(|b|^{2}+\operatorname{Im}^{2}(\bar{b} d)\right)$. Thus all of these inequalities are equivalent to

$$
\left(|b|^{2}+\operatorname{Im}^{2}(\bar{b} d)\right)\left((a c+\operatorname{Re}(\bar{b} d))^{2}+\left(\operatorname{Im}^{2}(\bar{b} d)-1\right)\right)>0 .
$$


This inequality holds since $\operatorname{Im}^{2}(\bar{b} d)>1$ by $(10)$, and so we have $\arg \left(z_{ \pm} / v\right)>0$, as desired.

This argument shows that if the planimeter is following either of the periodic trajectories, when the tracer point is at the initial vertex of the parallelogram the chisel edge is in the interior of the angle formed by the adjacent edges. It holds for the other vertices as well. Figure 10 illustrates this situation. As the tracer point moves along the initial edge of the parallelogram from 0 to $v$, the chisel edge follows a standard tractrix. It stays to the left of the direction of motion, that is, on the same side of the edge as the parallelogram, and thus only rotates counterclockwise. When the tracer point reaches $v$, the chisel edge is in the interior of the angle at $v$, and so is to the left of the new direction of motion when the tracer point starts moving from $v$ to $v+w$ (see Figure 11). This continues around the parallelogram, and so the planimeter has made a full rotation when the tracer point returns to the origin.

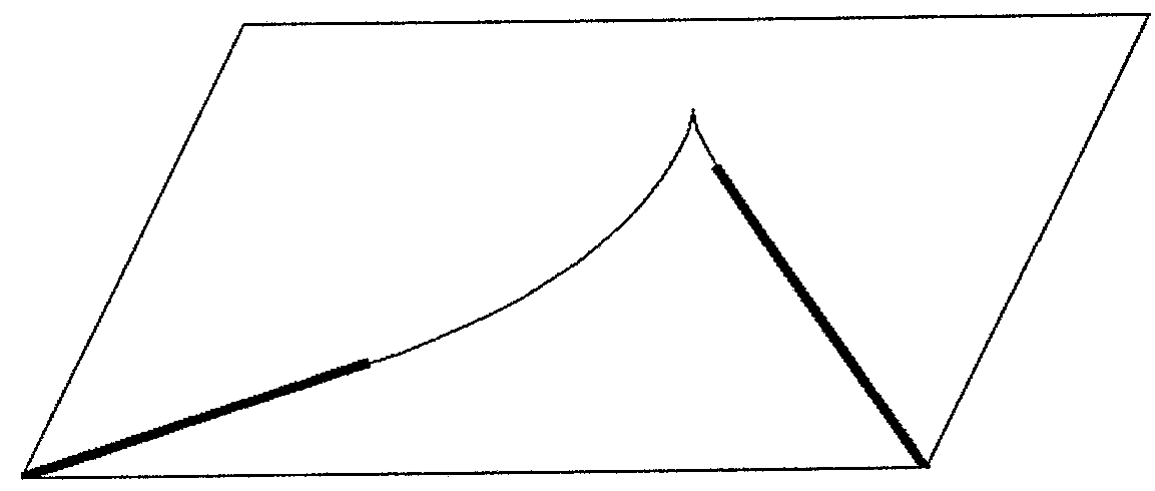

Figure 11.

Final Observations. Note that the condition which implies that the holonomy has an attracting fixed point is $\operatorname{Im}^{2}(\bar{b} d)>1$. As the proof shows, this happens for parallelograms with area exactly $\pi \ell^{2}$, and even for some with area slightly less. Under the assumption that $A \geq \pi \ell^{2}$, the minimum value of $\operatorname{Im}(\bar{b} d)=\sinh |\beta| \sinh |\delta| \sin \theta$ is achieved for the square of area $\pi \ell^{2}$.

It seems intuitively clear that $z_{+}$is the attracting fixed point and that $z_{-}$is the repelling fixed point. To confirm this, let $h(z)=H \cdot z$ for $z \in \mathbb{C}$. Since $h$ preserves $S^{1}$ and its orientation, $h^{\prime}\left(z_{+}\right)$and $h^{\prime}\left(z_{-}\right)$are both positive. A computation yields

$$
h^{\prime}\left(z_{ \pm}\right)=\left(2 \operatorname{Im}(\bar{b} d)\left(\operatorname{Im}(\bar{b} d) \pm \sqrt{\operatorname{Im}^{2}(\bar{b} d)-1}\right)-1\right)^{-2} .
$$

Remembering that $\operatorname{Im}(\bar{b} d)>1$, it follows that $h^{\prime}\left(z_{+}\right)<1$ and $h^{\prime}\left(z_{-}\right)>1$, justifying intuition.

As noted earlier, the results of Prytz and Hill imply that when the boundary of a small region is traced, the resulting holonomy has no fixed points. There are, however, short 
curves which result in holonomy with fixed points. As an example, let $v$ and $w$ be as in the proof (but without any assumption about the area of the parallelogram), and consider the path consisting of the parallelogram in the proof and its reflection through the origin. More specifically, the path is the polygonal "figure eight" formed by the successive vertices: $0, v, v+w, w,-w,-v-w,-v, 0$. Note that the oriented area bounded by this path is 0 . The resulting holonomy is $\tilde{H}=e^{X} e^{Y} e^{-X} e^{-2 Y} e^{-X} e^{Y} e^{X}$, where $X$ and $Y$ are given by (9). A computation shows that $\operatorname{tr} \tilde{H}=2+16 \operatorname{Im}^{2}(\bar{b} d)|a d+b c|^{2}$. As long as $v$ and $w$ are independent (which implies $\operatorname{Im}(\bar{b} d) \neq 0$ and $a d+b c \neq 0$ ) we have $\operatorname{tr} \tilde{H}>2$, and so $\tilde{H}$ has two fixed points. Furthermore, the fixed points are antipodal. This isn't surprising, given the symmetry of the "figure eight" about the base point 0 . (The expressions for the fixed points are very long and not very enlightening, so they are omitted.) Thus $\tilde{H}=e^{Z}$, where $Z=\omega(u)$ for some $u \in \mathbb{R}^{2}$. As we have seen, elements of $\mathfrak{s u}(1,1)$ of this form generate $\mathfrak{s u}(1,1)$. It follows that the holonomy group is generated by short loops.

\section{Figure Credits}

Figures 1(a,b) are from "Mathematical Machines" by Francis J. Murray [Mu, p. 348], Copyright (c) 1961 by Columbia University Press, and are reprinted with permission of the publisher. Figure 2 is from [Pou], with letters added. Figure 7 is from [G]. Animated versions of some of the figures are available on the author's web page (URL below).

\section{REFERENCES}

[Ba] G. Barnes, Hatchet or Hacksaw Blade Planimeter, Am. J. Physics 25 (1957), 25-29.

[Bo] W. M. Boothby, An Introduction to Differentiable Manifolds and Riemannian Geometry, Academic Press, New York, 1975.

[Cou] R. Courant, Differential and Integral Calculus, Vol. II, Nordemann, New York, 1934.

[Cox] H. S. M. Coxeter, Introduction to Geometry, second ed., Wiley, New York, 1989.

[Cr] A. R. Crathorne, The Prytz Planimeter, Am. Math. Monthly 15 (1908), 55-57.

[Fe] M. Fecko, Gauge-potential Approach to the Kinematics of a Moving Car, Il Nuovo Cimento B 111 (1996), 1315-1332.

[Fo1] R. L. Foote, A Plenitude of Planimeters, in preparation.

[Fo2] R. L. Foote, Planimeters and Isoperimetric Inequalities on Constant Curvature Surfaces, in preparation.

[G] J. Goodman (pub. anon.), Goodman's Hatchet Planimeter, Engineering, Aug. 21, 1896, $255-56$.

[He] O. Henrici, Report on Planimeters, British Assoc. for the Advancement of Science, Report of the 64th meeting (1894), 496-523.

[Hi] F. W. Hill, The Hatchet Planimeter, Philosophical Magazine, S. 5, Vol. 38, No. 232, Sept., 1894, 265-269; Proc. of the Physical Society 13, 229-234 (same paper appears twice).

[K] A. Kriloff, On the Hatchet Planimeter, Bulletin de l'Académie Impériale des Sciences de St. Pétersbourg, T. XIX, No. 4 \& 5, Nov/Dec, 1903, 221-227.

[KN] S. Kobayashi and K. Nomizu, Foundations of Differential Geometry, Vol. I, Wiley-Interscience, New York, 1963.

[KMS] I. Kolář, P. W. Michor, and J. Slovák, Natural Operations in Differential Geometry, SpringerVerlag, Berlin, 1993.

[L] D. N. Lehmer, Concerning the Tractrix of a Curve, with Planimetric Application, Annals of Math 13 (1899), 14-20. 
[Me] A. L. Menzin, The Tractigraph, an Improved form of Hatchet Planimeter, Engineering News, Vol. 56, No. 6 (1906), 131-132.

[Mo] F. Morley, The "No-Rolling" Curves of Amsler's Planimeter, Annals of Math 13 (1899), 21-30.

$[\mathrm{Mu}]$ F. J. Murray, Mathematical Machines, Vol. 2, Analog Devices, Columbia University Press, New York, 1961.

[Pe] Olaf Pedersen, The Prytz Planimeter, From Ancient Omens to Statistical Mechanics (J.L. Berggren and B.R. Goldstein, eds.), University Library, Copenhagen, 1987.

[Poo] W. A. Poor, Differential Geometric Structures, McGraw-Hill, 1981.

[Pou] A. Poulain, Les Aires des Tractrices et le Stang-Planimètre, J. de Mathématiques Spéciales, Vol. 4, No. 2 (1895), 49-54. (French)

[Pr1] H. Prytz (pseud. 'Z'), Stangplanimetret, Den Tekniske Forenings Tidsskrift 10 (1886), 23-28 (appendix to Heinrich Ohrt, Om Planimetre, 14-28). (Danish)

[Pr2] H. Prytz, The Hatchet Planimeter, (letter to the editor), Engineering 57 (June 22, 1894), 813.

[Pr3] H. Prytz, The Prytz Planimeter, (two letters to the editor), Engineering (September 11, 1896), 347.

[Pr4] H. Prytz, The Hatchet Planimeter and 'Tractigraph', (letter to the editor), Engineering News, Vol. 57, No. 14 (1907), 386.

[Sa] J. Satterly, The Hatchet Planimeter, J. Royal Astronomical Soc Canada, Vol. 15, No. 6 (1921), $221-243$.

[Sc] E. K. Scott, An Improved Stang Planimeter, Engineering (Aug. 14, 1896), 205-206.

[Se] C. L. Seigel, Topics in Complex Function Theory, Vol. II, Wiley-Interscience, New York, 1971.

[St] C. L. Strong, An Excursion into the Problem of Measuring Irregular Areas, Scientific American (The Amateur Scientist column), Vol. 199, No. 2 (1958), 107-114 (letter from F. W. Niedenfuhr).

Department of Mathematics \& Computer Science, Crawfordsville, IN 47933

E-mail address: footer@wabash.edu

Web page: http://persweb.wabash.edu/facstaff/footer/ 